\title{
Dynamik der Energiewende erhalten und erweitern
}

\section{NaWis \\ Verbund für Nachhaltige Wissenschaft}

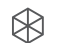

LEUPHANA

U N I K A S S E L

$\begin{array}{lllllllllll} & V & E & R & S & \mid & T & A & \end{array}$

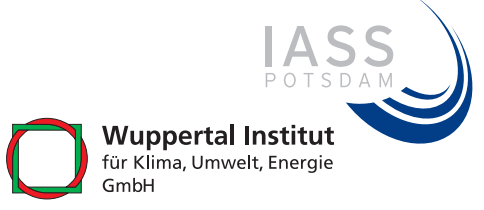

\author{
Dass die Energiewende überall in den Regionen erhebliche \\ Dynamiken und Innovationen hervorruft, hat einmal mehr das \\ Leuphana Energieforum 2014 gezeigt.
}

Trotz des politischen Gegenwinds bleiben

die Bürgerenergieakteure extrem wichtig

für die Akzeptanz der Energiewende

und deren erfolgreiche Ausdehnung auf

Wärmebereitstellung und Verkehr.

Thomas Schomerus, Erik G. Hansen, Stefan Lechtenböhmer
$D^{i}$ ie Energiewende muss sich ständig wandelnden Herausforderungen anpassen. Ursprünglich war sie als Gegenbewegung zum Ausbau der Atomenergie nach der ersten Ölpreiskrise in den 1970er Jahren entstanden; gefordert war damals, die Energiewende durch Wachstum und Wohlstand ohne Erdöl und Uran zu bewerkstelligen (Krause et al. 1980) ${ }^{1}$; heute umfasst sie weitere Themen:

1. den durch fossile Kraftwerke forcierten Klimawandel,

2. ökonomische Aspekte; beispielsweise betonte Klaus Töpfer im Rahmen des Leuphana Energieforums 2014, dass Europa allein aus Kostengründen zu einer Erneuerbare-Energien(EE)-Region werde $^{2}$; und

3. die Aussicht auf langfristige Unabhängigkeit von endlichen fossilen Ressourcen sowie - angesichts aktueller zwischenstaatlicher Spannungen wie dem Ukraine/Russland-Konflikt - die Verminderung der Abhängigkeit von fossilen Energieimporten.

1 Siehe auch www.energiewende.de/index.php?id=5. 2 Informationen zum Leuphana Energieforum 2014 und Download von Präsentationen unter www.leuphana.de/partner/regional/aktuell/ veranstaltungen/energieforum/energieforum2014.html.

\section{Einblicke in das Leuphana Energieforum 2014}

Das Leuphana Energieforum 2014 hat sich einigen dieser Themen gestellt und sowohl ihre Relevanz für die Energiewirtschaft in der Nordregion als auch die möglichen Lösungsbeiträge aus der Region beleuchtet. Rund 260 Teilnehmer(innen) aus der vor allem von Bürgerenergie geprägten regionalen Energiewirtschaft, aus Hochschulen und Politik besuchten das Forum, das zum dritten Mal stattfand.

Kooperationspartner sind neben der IHK Lüneburg-Wolfsburg auch der Verbund für Nachhaltige Wissenschaft (NaWis), dessen Mitglieder jeweils eigene Beiträge zum Programm beisteuerten. Das Forum hat sich als regionale Plattform der Energiewende etabliert. Es dient dem Wissenstransfer zwischen Wissenschaft, Wirtschaft und Zivilgesellschaft, der Vernetzung untereinander sowie der Formulierung von Forderungen aus der Region an die politisch für die Umsetzung der Energiewende Verantwortlichen.

Die Energiewende erfordert regionale und lokale Lösungen. Große Zustimmung beim Energieforum erntete zum Beispiel Wolfgang Schmalz, Geschäftsführer der J. Schmalz GmbH in Glatten/Schwarzwald sowie Gewinner des europäischen Green-Blue-Energy Factory-Award (GBE Fac- tory 2014), der für sein Unternehmen im Bereich der Vakuumtechnik über den eigenen Verbrauch hinaus Energie selbst produziert und somit selbst von einem „Positiv-Energie-Unternehmen“ spricht (Schmalz 2013). Solche „Pioniere des Wandels“ (WBGU 2011) brauchen verlässliche

Kontakt Autoren: Prof. Dr. Thomas Schomerus | Professur für öffentliches Recht, insbesondere Energie- und Umweltrecht | E-Mail: schomerus@uni.leuphana.de

Prof. Dr. Erik G. Hansen | Gastprofessur Management der Energiewende | Innovations-Inkubator und Centre for Sustainability Management (CSM) | E-Mail: erik.hansen@inkubator.leuphana.de beide: Leuphana Universität Lüneburg | Lüneburg | Deutschland

Dr. Stefan Lechtenböhmer | Forschungsgruppe Zukünftige Energie- und Mobilitätsstrukturen | Wuppertal Institut für Klima, Umwelt, Energie | Wuppertal | Deutschland | E-Mail:

stefan.lechtenboehmer@wupperinst.org

Kontakt NaWis-Runde: Prof. Dr. Uwe Schneidewind | Wuppertal Institut für Klima, Umwelt, Energie GmbH | Döppersberg 19 | 42103 Wuppertal | Deutschland | Tel.: +492022492100|

E-Mail: uwe.schneidewind@wupperinst.org | www.wupperinst.org

(C) 2014 T. Schomerus et al.; licensee oekom verlag. This is an article distributed under the terms of the Creative Commons Attribution License (http://creativecommons.org/licenses/by/3.0), which permits unrestricted use, distribution, and reproduction in any medium, provided the original work is properly cited. 
Rahmenbedingungen. Ob die aktuellen politischen und rechtlichen, aus Brüssel und Berlin kommenden Weichenstellungen insoweit ausreichen, ist zweifelhaft.

\section{Energiewende in der aktuellen politischen Lage}

Aktuelle Entwicklungen, wie das Erneuerbare-Energien-Gesetz (EEG) 2014, setzen Fragezeichen, ob sich die unverändert bestehenden Ziele auch weiterhin in Handlungen niederschlagen werden. Die Neuerungen des aufgrund einer europarechtlich sehr fragwürdigen Kommissionsvorgabe (Münchmeyer et al. 2014) im August 2014 in Kraft getretenen EEG waren daher eines der zentralen Themen des Energieforums. Diskutiert wurden vor allem die Einführung einer Mengensteuerung für die Onshore-Windenergie mit einem ,atmenden Deckel“, der faktische Stopp des Baus von neuen Bioenergieanlagen durch den Ausbaupfad von nur noch 100 Megawatt pro Jahr, die teilweise Erhebung der EEG-Umlage für den Eigenverbrauch (gilt auch für Kraftwärmekopplung und sogar für Solarstrom), die stufenweise Einführung der verpflichtenden Direktvermarktung und das ab 2017 vorgesehene Ausschreibungsverfahren zur Ermittlung der Vergütungshöhe.

Insbesondere das Ausschreibungsverfahren - vor allem die Frage, ob von Bürgerenergie getragene Projekte in dessen Rahmen eine Zukunft haben - weckt erhebliche Befürchtungen (Degenhart und Nestle 2014). Hinter diesen Reformen lässt sich eine Strategie der Bundesregierung vermuten, die bei Anteilen von 30 Prozent und mehr EE an der Stromversorgung darauf setzt, dass große, zentrale Akteure verloren gegangenes Terrain zurückgewinnen sollen. Ein solches mangelndes Vertrauen in die Bürgerenergie gefährdet nicht nur regionale Arbeitsplätze, sondern auch die Initiative für den Ausbau der EE und deren Akzeptanz (Maly et al. 2014).

Dass die EEG-Umlage, vor allem als Folge des bereits im EEG 2012 eingeführten „atmenden Deckels“ für die Solarenergie, erstmals sinken wird (um 0,07 Cent auf 6,17 Cent pro Kilowattstunde 2015; Bundesnetzagentur 2014), erscheint als ein Erfolg für die von der Politik intendierte „Kosten- bremse“. Doch angesichts der erforderlichen Investitionen in die Verteilnetze und die dadurch steigenden Netzentgelte wird es kaum zu Entlastungen für die Verbraucher(innen) kommen (BDEW 2014b). Abgesehen davon ist die Umlage nur teils durch die Kosten der Stromerzeugung aus EE bedingt (und betrifft hier weitgehend nur finanzielle Altlasten der bestehenden Anlagen und kaum den Ausbau mit Neuanlagen; vergleiche Töpfer und Bachmann 2013), zum anderen Teil fließt zum Beispiel die besondere Ausgleichsregelung für energieintensive Unternehmen (Öko-Institut 2013) ein.

Ein Scheuklappenblick auf die Kosten allein wird der Bedeutung der Energiewende für die nachhaltige Entwicklung nicht gerecht und von den meisten Bürger(inne)n auch nicht geteilt (AEE 2014).

\section{Von der Stromwende zur Energiewende}

Bislang fokussierte die Energiewende zu sehr auf die Erfolgsgeschichte des steigenden Anteils von EE in der Stromversorgung, der (vor allem aufgrund der im ursprünglichen $E E G$ angelegten garantierten Einspeisevergütung - die nun aber zunehmend erodiert) im ersten Halbjahr 2014 rund 28 Prozent erreichte (BDEW 2014a). Nur ein Fünftel des Endenergieverbrauchs in Deutschland allerdings ist Strom - dagegen wird etwa die Hälfte zur Erzeugung von Prozess- und Raumwärme sowie ein Drittel im Verkehrsbereich als Kraftstoff verbraucht; der Anteil EE an diesem Gesamtendenergieverbrauch stagniert seit 2012 auf dem konstant niedrigem Niveau von nur 12,3 Prozent. ${ }^{3}$

Die Botschaft, die auch vom Energieforum 2014 ausging, ist damit eindeutig: Die Dynamik der Energiewende muss auf die ganze Breite des Energieverbrauchs ausgedehnt werden. Das pauschale Abwürgen der vielseitig einsetzbaren Bioenergie durch das EEG 2014 und der fehlende Ausbau der Förderung alternativer ausgereifter Wärmetechnologien wie der Solarthermie sind daher Schritte in die falsche Richtung und belasten die nachhaltige Entwicklung vor allem auch ländlicher Regionen.

Letztendlich fehlen Instrumente mit der Schlagkraft des originalen $E E G$ für die
Energiewende als Ganzes respektive der Sektoren Wärme/Gebäude und Verkehr, was sich auch in der jüngsten Trendbewertung der Europäischen Umweltagentur (EEA) (2014) widerspiegelt: Bei der Minderung des Energiebedarfs ist Deutschland als eines von nur drei EU-Ländern nicht im Zielkorridor. Dies wirkt sich auf die Klimaschutzerfolge in Haushalten, Gewerbe und Verkehr aus, die ebenfalls mit negativer Tendenz außerhalb des Zielkorridors bis 2020 liegen. Hier fehlt wohl auch der politische Durchsetzungswille. So verzichtete die Bundesregierung darauf, zur Umsetzung der Energieeffizienzrichtlinie (RL 2012/ 27/EU) für ein Energieeffizienzverpflichtungssystem zu optieren, das dem $E E G$ in seiner potenziellen Wirksamkeit vergleichbar gewesen wäre (Schomerus 2013). Stattdessen sollen alternative Maßnahmen umgesetzt werden (dena 2013).

Im Gegensatz dazu wären jedoch innovative und mutige Instrumente vonnöten, die insbesondere das Energienutzungsverhalten der einzelnen Verbraucher(innen) adressieren und letztendlich verändern. Ein gutes Beispiel ist das dänische Modell des Verbots neuer Öl- und Gasheizungen (MCEB 2012).

\section{Bürger(innen) und Unternehmer(innen) als Treiber der Energiewende}

An guten Vorschlägen für eine erfolgreiche Weiterentwicklung der Energiewende mangelt es also nicht. Der Wille zum Umdenken kann aber nicht ausschließlich von oben kommen, sondern muss weiter von unten - den Bürger(inne)n und der regionalen mittelständischen Wirtschaft - getragen werden. So können beispielsweise Bürgergenossenschaften die Diffusion der EE wesentlich vorantreiben (Degenhart und Nestle 2014). Die Bürger(innen) können auch in der Rolle als Unternehmer(innen) - vor allem kleiner und mittelständischer oder auch kommunaler Unternehmen die Energiewende beschleunigen (Hansen 2014): Effizienzverbesserungen und klassisches Energiemanagement (Hansen und Klewitz 2012), Energieautarkiebestrebungen (McKenna et al. im Erscheinen) und

3 www.umweltbundesamt.de/daten 
sogar die Entwicklung zum „Positiv-Energie-Unternehmen“ (GBE Factory 2014) fördern die dezentrale Diffusion der EE und ermöglichen den Unternehmen, Wettbewerbsvorteile daraus zu generieren - zum Beispiel durch langfristige Energiekostenreduktion oder Imagesteigerung. Ähnliches gilt, wie viele Beispiele zeigen, auch für Dörfer und Städte. Durch eigenes, auch unternehmerisches, Engagement kann die Energieversorgung grüner, günstiger und mit gesteigerter lokaler Wertschöpfung umgestaltet werden. Die Vorreiter sind neben der Stromerzeugung bereits in der regenerativen Wärmeversorgung aktiv und erschließen außerdem zum Beispiel das Thema (Elektro-)Mobilität.

Das Leuphana Energieforum 2014 hat einerseits gezeigt, dass die Bürgerenergie angesichts der derzeitigen Entwicklungen zwar Gefahr läuft, als Treiber der Energiewende ins Hintertreffen zu geraten, andererseits aber auch sehr deutlich gemacht, dass die notwendige Dynamik nur erhalten und über die Stromerzeugung hinaus auf andere Sektoren ausgedehnt werden kann, wenn dies nach wie vor durch eine breite Bürgerenergiebewegung getragen wird.

\section{Literatur}

AEE (Agentur für Erneuerbare Energien). 2014 92 Prozent der Deutschen wollen den Ausbau Erneuerbarer Energien. Pressemitteilung 15.10.2014. Berlin: AEE.

BDEW (Bundesverband der Energie- und Wasserwirtschaft). 2014 a. Erneuerbare Energien erreichen neuen Rekordwert. Pressemitteilung 24.07.2014. Berlin: BDEW.

BDEW. 2014b. Unterschiedliche Faktoren beeinflussen Strompreise. Pressemitteilung 15.10.2014. Berlin: BDEW.

Bundesnetzagentur. 2014. EEG-Umlage beträgt im kommenden Jahr $6,17 \mathrm{ct} / \mathrm{kWh}$. Pressemitteilung 15.10.2014. Bonn: Bundesnetzagentur.

Degenhart, H., U. Nestle. 2014. Marktrealität von Bürgerenergie und mögliche Auswirkungen von regulatorischen Eingriffen. www.bund.net/ fileadmin/bundnet/pdfs/klima_und_energie/ 140407_bund_klima_energie_buergerenergie_ studie.pdf (abgerufen 28.10.2014).

dena (Deutsche Energie-Agentur). 2013. Steigerung der Energieeffizienz mit Hilfe von EnergieeffizienzVerpflichtungssystemen. Berlin: dena. www.dena.de/fileadmin/user_upload/Presse/ studien_umfragen/Energieeffizienz-Verpflichtungssysteme/Studie_EnergieeffizienzVerpflichtungssysteme_EnEffVSys.pdf (abgerufen 28.10.2014).
EEA (European Environment Agency). 2014. Trends and projections in Europe 2014. EEA Report 6/2014. Kopenhagen: EEA.

EEG (Erneuerbare-Energien-Gesetz). 2012. Gesetz zur Neuregelung des Rechtsrahmens für die Förderung der Stromerzeugung aus erneuerbaren Energien vom 28. Juli 2011. Bundesgesetzblatt, Teil I, 42: 1634

EEG. 2014. Gesetz zur grundlegenden Reform des Erneuerbare-Energien-Gesetzes und zur Änderung weiterer Bestimmungen des Energiewirtschaftsrechts vom 21. Juli 2014. Bundesgesetzblatt, Teil I, 33: 1066

GBE Factory (Green Blue Energy Factory). 2014. Green Blue Energy Factory: Final Report. Venice: GBE Factory.

Hansen, E. G. 2014. Energiewende trifft Unternehmertum. Magazin der Industrie- und Handelskammer Stade 6: 20.

Hansen, E. G., J. Klewitz. 2012. The role of an SME's green strategy in public-private ecoinnovation initiatives: The case of ecoprofit. Journal of Small Business and Entrepreneurship 25/4: 451-477.

Krause, F., H. Bossel, K.-F. Müller-Reissmann. 1980. Energie-Wende. Wachstum und Wohlstand ohne Erdöl und Uran. Ein Alternativ-Bericht des Öko-Institut, Freiburg. Frankfurt am Main: S. Fischer.

Maly, C., M. Meister, T. Schomerus. 2014. EEG 2014 - Das Ende der Bürgerenergie? Energierecht 4/14: 147-154.

MCEB (Ministry of Climate, Energy and Building) 2012. Accelerating green energy towards 2020: The Danish energy agreement of March 2012. Kopenhagen: MCEB.

McKenna, R., T. Jäger, W. Fichtner. Im Erscheinen. Energieautarkie - ausgewählte Ansätze und Praxiserfahrungen im deutschsprachigen Raum. uwf UmweltWirtschaftsForum.

Münchmeyer, H., M. Kahles, F. Pause. 2014 Erfordert das europäische Beihilferecht die Einführung von Ausschreibungsverfahren im EEG? Würzburg: Stiftung Umweltenergierecht.

Öko-Institut. 2013. Analyse der EEG-Umlage 2014. Berlin: Öko-Institut.

RL 2012/27/EU. Richtlinie 2012/27/EU des europäischen Parlaments und des Rates zur Energieeffizienz vom 25. Oktober 2012. Amtsblatt der EU L 315: 1-56.

Schmalz. 2013. Nachhaltigkeitsbericht - Schmalz ecoSYSTEM. Glatten: J. Schmalz GmbH.

Schomerus, T. 2013. Die Umsetzung von Art. 7 Energieeffizienz-Richtlinie 2012/27/EU in nationales Recht - Energieeffizienzverpflichtungssysteme oder andere strategische Maßnahmen? EnergieRecht 2/5: 184-191.

Töpfer, K., G. Bachmann. 2013. Kostenschnitt für die Energiewende. Die Neuordnung der Stromkosten ist die Voraussetzung für die Reform der Energiepolitik. Berlin: Institute for Advanced Sustainability Studies (IASS).

WBGU (Wissenschaftlicher Beirat der Bundesregierung Globale Umweltveränderungen). 2011. Welt im Wandel - Gesellschaftsvertrag für eine Große Transformation. Hauptgutachten. Berlin: WBGU. 